\title{
Effect of hyphal micromorphology on bioreactor performance of antibiotic-producing Saccharopolyspora erythraea cultures
}

\author{
Steven M. Martin† and Michael E. Bushell \\ Author for correspondence: Michael E. Bushell. Tel: +44 1483 259277. Fax: +441483 300374. \\ e-mail: bss1mb@surrey.ac.uk
}

Microbial Physiology Group, School of Biological Sciences, University of Surrey, Guildford, Surrey GU2 5XH, UK

\begin{abstract}
When Saccharopolyspora erythraea biomass from submerged culture was filtered (100-120 $\mu \mathrm{m}$ sintered glass filter) the antibiotic yield of the retentate (mean hyphal particle diameter $103 \mu \mathrm{m})$ was significantly higher [1.740 $\mathrm{mg}$ ( $\mathrm{mg}$ biomass) $\left.{ }^{-1}\right]$ than that of the filtrate [1.286 $\mathrm{mg}^{\left(\mathrm{mg}^{2} \text { biomass) }\right.}{ }^{-1}$; mean hyphal particle diameter $88 \mu \mathrm{m}$ ]. A hypothesis to explain this is that there is a critical hyphal particle diameter, below which the particle is incapable of producing antibiotic. This would be a consequence of the site of antibiotic production occurring at a fixed distance from the growing hyphal tip. A protocol is proposed for calculation of the hypothetical critical hyphal diameter $(88 \mu \mathrm{m}$ in this case). The proportion of retentate (more productive) fraction of biomass varied between $60 \%$ and $30 \%$ during the course of a batch culture. Bioreactor stirrer speed significantly affected mean hyphal particle diameter $(70 \mu \mathrm{m}$ at 1500 r.p.m.; $124 \mu \mathrm{m}$ at 750 r.p.m.) and antibiotic productivity [0.867 mg (mg biomass) ${ }^{-1}$ at 1500 r.p.m.; 0.913 mg (mg biomass) ${ }^{-1}$ at 750 r.p.m.].
\end{abstract}

Keywords: hyphal micromorphology, bioreactor performance, secondary metabolism, antibiotic production, erythromycin

\section{INTRODUCTION}

We have reported that a significant hyphal breakage rate occurs in shake-flask liquid cultures of many Streptomyces species, a phenomenon that accounts for these species producing antibiotics on agar but not in liquid culture (Pickup et al., 1993). We obtained evidence that resistance to hyphal breakage is dependent on the activity of a peptidoglycan-synthesizing enzyme, phospho- $N$ acetylmuramyl pentapeptide translocase, whose activity appears to determine hyphal tensile strength (Pickup \& Bushell, 1995). Hyphal breakage appears to result in the formation of viable mycelial fragments, incapable of antibiotic production, whose growth rates are equal to those of the unbroken hyphal units from which they were derived (Pickup et al., 1993).

Other laboratories have described correlations between product formation and morphology in cultures of Streptomyces species, reporting lack of production in cultures whose micromorphologies appeared fragmented (Shomura et al., 1979; Roth et al., 1982). Reports have also appeared relating antibiotic production to hyphal ultra-

†Present address: Xenova Group PLC, 240 Bath Road, Slough SL1 4EQ, UK. structure (Williams et al., 1974; Bewick \& Williams, 1977; Bewick et al., 1977; Liu et al., 1984) but it has not been possible to relate these to fragmentation phenomena.

Our observations suggest that hyphal particles with a range of sizes coexist in submerged liquid culture of streptomycete species and that the antibiotic yield of these cultures is dependent on the ratio of the number of larger (antibiotic-producing) hyphal fragments to that of the smaller (non-producing) fragments.

\section{METHODS}

Strains and culture media. Saccharopolyspora erythraea NRRL 2338 was used throughout. Antibiotic concentrations were measured routinely by bioassay using a strain of Artbrobacter citreus (GL1) obtained from the Shell Laboratories culture collection, Sittingbourne, Kent, UK. The chemically defined (nitrogen-limited) antibiotic production medium for $S$. erytbraea contained the following major nutrients $\left(\mathrm{g} \mathrm{l}^{-1}\right.$ in reverseosmosis-purified water): glucose, $15 ; \mathrm{NaNO}_{3}, 1 \cdot 19 ; \mathrm{KH}_{2} \mathrm{PO}_{4}$, $3.0 ; \mathrm{K}_{2} \mathrm{HPO}_{4}, 7 \cdot 0$; and the following trace components $\left(\mathrm{g} \mathrm{l}^{-1}\right)$ : $\mathrm{MgSO}_{4} \cdot 7 \mathrm{H}_{2} \mathrm{O}, 0.25 ; \mathrm{FeSO}_{4} \cdot 7 \mathrm{H}_{2} \mathrm{O}, 0.025 ; \mathrm{CuCl}_{2}, 0.00053$; $\mathrm{CoCl}_{2}, 0.00055 ; \mathrm{CaCl}_{2} .2 \mathrm{H}_{2} \mathrm{O}, 0.0138 ; \mathrm{ZnCl}_{2}, 0.0104 ; \mathrm{MnCl}_{2}$, $0.0062 ; \mathrm{Na}_{2} \mathrm{MoO}_{4}, 0.0003$. Glucose, salts and trace solution were autoclaved separately and then mixed. The $\mathrm{pH}$ was adjusted to $7 \cdot 0$ with $5 \mathrm{M} \mathrm{KOH}$ prior to autoclaving.

S. erythraea was maintained on tomato purée oatmeal agar $\left(\mathrm{g} \mathrm{l}^{-1}\right.$ : 
tomato purée, 10 ; oatmeal, 10 ; agar, 20) and $A$. citreus was maintained on nutrient agar. Both were grown in $250 \mathrm{ml}$ baffled Erlenmeyer flasks containing $25 \mathrm{ml}$ nutrient broth at $30^{\circ} \mathrm{C}$ on a rotary shaker at 250 r.p.m. After $48 \mathrm{~h}$ agitation ( $S$. erythraea), $2 \mathrm{ml}$ was removed and used to inoculate preculture flasks containing $23 \mathrm{ml}$ defined medium. After $48 \mathrm{~h}$ further incubation, the precultures were used at approximately $5 \%(\mathrm{v} / \mathrm{v})$ as an inoculum for flask or bioreactors.

Bioreactor culture. An LH 2000 series bioreactor with a working volume of $7 \mathrm{l}$ was used. Agitation was provided by disc turbine impellers, rotating at 500 r.p.m. (batch), 750 r.p.m. (chemostat low speed) or 1500 r.p.m. (chemostat high speed) and sterile air was supplied through a sparger at a flow rate of $0.8 \mathrm{v} / \mathrm{v} / \mathrm{m}$. The temperature was controlled at $30^{\circ} \mathrm{C}$. Bioreactor dissolved oxygen concentration was monitored using an Ingold polarographic dissolved oxygen electrode. The $\mathrm{pH}$ was monitored throughout but was not controlled. The cultures, which contained a phosphate buffering system (see above) increased in $\mathrm{pH}$ over the course of an experiment by no more than 0.5 of a $\mathrm{pH}$ unit. Foaming was eliminated by including $0.01 \%$ Breox FM'T30 antifoam (Water Management and Gamlen) in the culture medium.

Chemostat cultures were performed using a peristaltic pump to introduce fresh medium into the vessel. A constant volume was maintained using a weir overflow pipe. A steady state was assumed when constant biomass concentration $( \pm 5 \%$ ) was observed in four samples, taken at the rate of three per mean culture residence time.

Antibiotic assays. A bioassay employing Artbrobacter citreus was performed using procedures described previously (Huck et al., 1991). Challenge strain seed cultures and assay plates were incubated at $30^{\circ} \mathrm{C}$, and the diameters of the zones of inhibition were recorded after $24 \mathrm{~h}$. Examination of a number of samples using high-performance liquid chromatography (Tsuji \& Goetz, 1978) confirmed that the bioassay values corresponded to erythromycin concentrations.

Residual glucose, nitrate and phosphate. A glucose-oxidasebased assay kit (Trinder system, Sigma) and a nitrate-reductasebased assay kit (Boehringer Mannheim) were employed. The colorimetric procedure described previously (McDermott $e t$ al., 1993) was used for phosphate detemination.

Culture filtration. A modification of the method described by Pickup \& Bushell (1995) was used. Sintered glass filters (pore size $100-120 \mathrm{~mm}$ ) were used instead of the muslin filters described by Pickup \& Bushell (1995). The filters were mounted in autoclavable glass and plastic holders and asepsis was maintained throughout the filtration. Prior to filtration, $400 \mathrm{ml}$ samples of culture were removed at regular intervals during batch fermentation and diluted with $100 \mathrm{mM}$ MOPS buffer $\left(\mathrm{pH} \mathrm{7.0)}\right.$ to give a final biomass concentration of $0.5 \mathrm{~g} \mathrm{l}^{-1}$.

Replacement culture experiments. Following filtration of a $10 \mathrm{~h}$ bioreactor sample, retentate, filtrate and unfiltered (centrifuged) mycelium were reinoculated into culture supernatant at $1 \mathrm{~g} \mathrm{l}^{-1}$. A control flask consisting of uninoculated supernatant was also incubated.

Reproducibility and statistical analysis. All experimental data presented in this paper were obtained from single cultures. Experiments were carried out in triplicate to ensure that the trends and relationships observed in the culture parameters measured were reproducible. Individual assays were replicated fourfold. Sampling shaker cultures involved sacrificing two cultures for each time point and assaying the contents in-

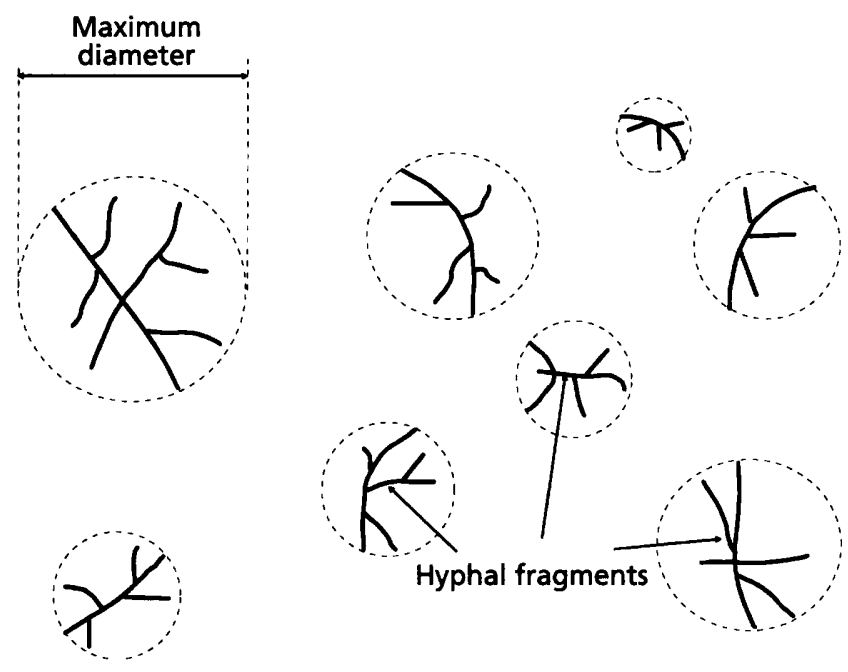

Fig. 1. Method used to estimate maximum diameter of hyphal fragments.

dividually. Experiments were rejected where a chi-squared test indicated significant differences between replicates. Biomass concentrations varied by no more than $5 \%$ between replicates. Error bars are not shown as they were mostly too small to be visible on the figures presented.

The Fisher-Berens test (Campbell, 1974) was used when it was necessary to determine whether two sets of data were significantly different. This requires the calculation of the $C$ statistic where:

$C=\frac{\bar{x}_{1}-\bar{x}_{2}}{\sqrt{\left(\frac{\sigma_{1}^{2}}{n_{1}}-\frac{\sigma_{2}^{2}}{n_{2}}\right)}}$

and $\bar{x}, \sigma$ and $n$ are mean, sample deviation and sample size of the two populations. The calculated value of $C$ is compared with those in an appropriate statistical table in order to test the null hypothesis that there is no difference between samples.

Hyphal fragment size measurement. A modification of the technique of Packer \& Thomas (1990) was used to prepare slides for analysis. Culture samples were diluted 50 -fold in $100 \mathrm{mM}$ MOPS buffer ( $\mathrm{pH} \mathrm{7.0)}$ ) and agitated to give a homogeneous suspension of mycelium. Samples of the suspension $(40 \mu \mathrm{l})$ were spread over the surface of the slide and air dried. When dried, the slides were stained with methylene blue solution $[0.3 \mathrm{~g}$ methylene blue, $30 \mathrm{ml} 95 \%(\mathrm{v} / \mathrm{v})$ methanol in $100 \mathrm{ml}$ water] for $1 \mathrm{~min}$. The slides were rinsed in water for $2 \mathrm{~min}$ and allowed to air dry for $2 \mathrm{~h}$. Six slides were prepared for each sample. The slides were examined and photographed using a Leitz orthoplan microscope with phase-contrast optics and a magnification of 200 . Sufficient fields were photographed to allow 250-300 separate fragments to be measured and counted. Photographs were examined and maximum particle diameter estimated using a stencil containing circles whose diameters ranged from 6 to $42 \mathrm{~mm}$, with an interval of $2 \mathrm{~mm}$. The scale was such that a length of $20 \mathrm{~mm}$ on the photograph corresponded to $100 \mu \mathrm{m}$. The maximum fragment diameter was defined as the smallest circle on the stencil into which a fragment could be completely fitted (Fig. 1). 


\section{RESULTS AND DISCUSSION}

\section{Antibiotic production during nitrogen-limited batch culture}

Production kinetics similar to those obtained previously (McDermott et al., 1993; Wilson \& Bushell, 1995) were observed. Culture biomass and antibiotic concentrations increased in parallel (Fig. 2), an observation that contrasts with the growth-dissociated production observed in carbon-limited cultures (McDermott et al., 1993; Lynch \& Bushell, 1995).

\section{Resolution of the culture into two fractions by filtration}

Filtration using the sintered glass filter resulted in two populations of hyphal particles, with mean particle diameters of $88 \mu \mathrm{m}$ (filtrate) and $102 \mu \mathrm{m}$ (retentate) (Fig. 3 ). Frequency analysis indicated that the particle size distributions overlapped (Fig. 3), with the diameter at peak frequency being significantly smaller in the filtrate than in the retentate (Fig. 3, Table 1).

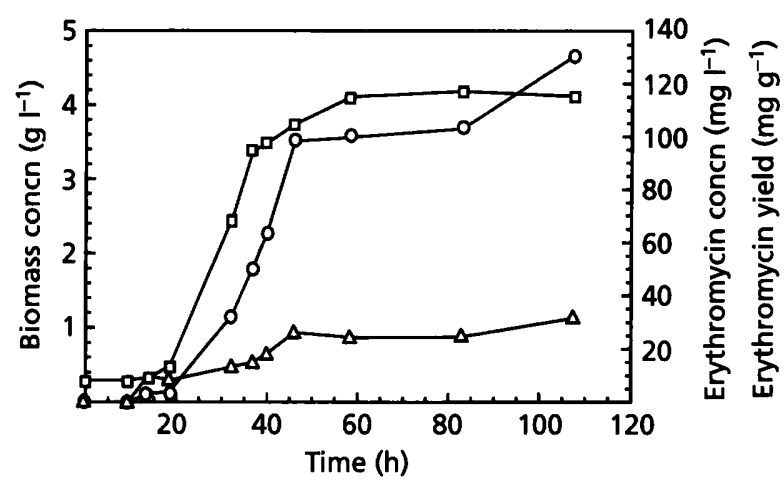

Fig. 2. Erythromycin concentration (O), biomass concentration ( $\square$ ) and erythromycin yield [mg erythromycin produced ( $g$ biomass) ${ }^{-1}$, since inoculation] $(\Delta)$ in nitrogen-limited batch bioreactor culture.

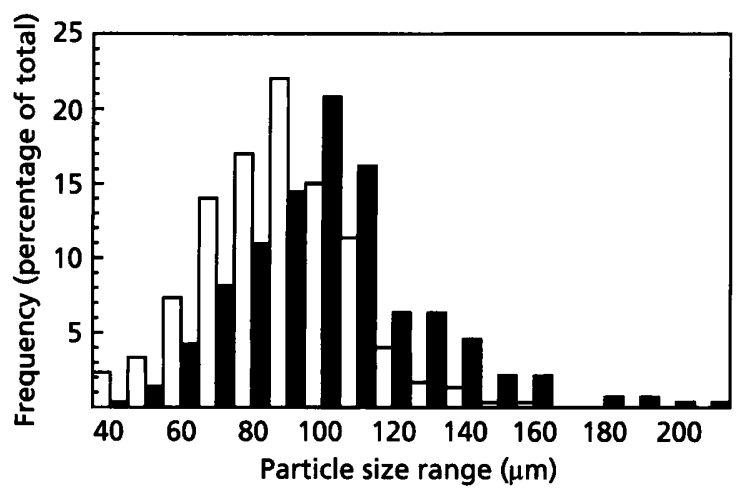

Fig. 3. Distribution histograms for particle size in the filtrate $(\square)$ and retentate $(\square)$ for a $24 \mathrm{~h}$ nitrogen-limited bioreactor culture.
Table 1. Statistical analysis of the hyphal particle size distribution data obtained on filtering the culture through a sintered glass filter

$C=7 \cdot 354$, for the two sets of particle size data; therefore reject null hypothesis that there is no difference between particle size distribution in retentate and filtrate $(P=0 \cdot 01)$.

\begin{tabular}{|lcc|}
\hline Statistic & Filtrate & Retentate \\
\hline Sample deviation $(\mu \mathrm{m})$ & $20 \cdot 86$ & $27 \cdot 04$ \\
Sample mean $(\mu \mathrm{m})$ & $88 \cdot 00$ & $102 \cdot 73$ \\
Variance $(\mu \mathrm{m})$ & 86.59 & $145 \cdot 71$ \\
Sample size & 300 & 284 \\
\hline
\end{tabular}

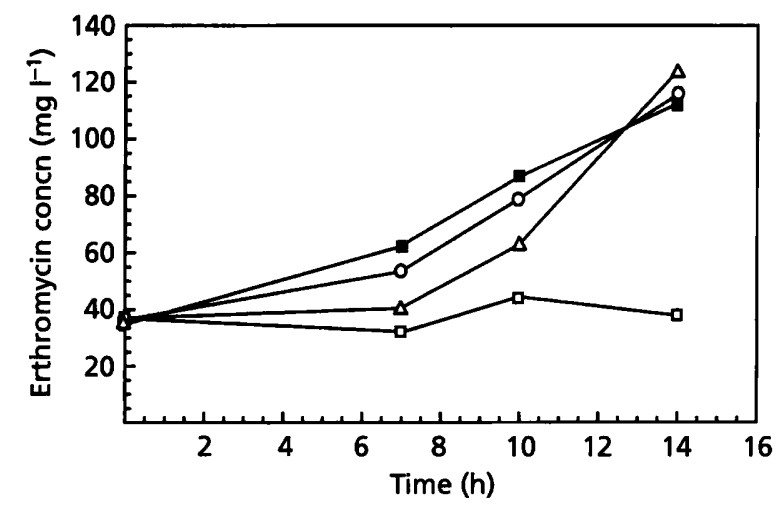

Fig. 4. Erythromycin concentrations obtained when culture filtrate $(\triangle)$, culture retentate $(\square)$ and unfiltered centrifuged mycelium $(O)$ were resuspended in culture supernatant and incubated. $\square$, Uninoculated control.

\section{Antibiotic production in the filtrate and retentate fractions}

During the replacement culture experiment, the erythromycin production potential of the two fractions was monitored at regular intervals for $14 \mathrm{~h}$ (Fig. 4). During the first $10 \mathrm{~h}$ the erythromycin titre in the retentatederived culture was higher than that observed in the filtrate-derived culture. This relationship between particle size fraction and antibiotic production appeared to break down as the culture incubation time increased, presumably because elongation and fragmentation took place during the subsequent incubation, resulting in loss of homogeneity of hyphal morphology. If baffled flasks were used to incubate the two fractions, the relationship between filtration and antibiotic production broke down more rapidly (within $3 \mathrm{~h}$ ), presurnably due to an increased rate of fragmentation.

\section{Specific erythromycin production rate}

The replacement culture experiment was repeated over $12 \mathrm{~h}$ and the biomass concentrations of the two cultures estimated so that specific production rate of the retentateand filtrate-derived cultures could be measured. The 
retentate productivity was $32 \%$ higher than that of the filtrate (specific erythromycin production rate at $12 \mathrm{~h}$ 1.74 versus $1.29 \mathrm{mg} \mathrm{g}^{-1} \mathrm{~h}^{-1}$ ), providing clear evidence that the larger hyphal fragments had a higher specific production rate than that of the smaller fragments; this is consistent with the notion that there are more antibiotic producing sites per unit hyphal mass in the larger particles.

\section{Calculation of the minimum hyphal particle size needed to support antibiotic production}

If it is assumed that there is a threshold hyphal particle size, below which particles are incapable of producing antibiotic (hypothetical), then the culture biomass consists of a proportion of non-producing biomass (small fragments) and a proportion of producing biomass (large fragments). If it is also assumed that the yield $(\Delta P / \Delta X)$ of the producing biomass is the same in retentate and filtrate, then the different production capabilities of the two fractions simply reflect the proportion of producing biomass in each fraction.

Let $A=$ weight of producing biomass in $1 \mathrm{~g}$ filtrate, $C=$ weight of producing biomass in $1 \mathrm{~g}$ retentate, $B=$ weight of non-producing biomass in $1 \mathrm{~g}$ filtrate, and $D=$ weight of non-producing biomass in $1 \mathrm{~g}$ retentate.

Antibiotic yield in the filtrate $=\frac{A \cdot x}{A+B}$

[g erythromycin ( $\mathrm{g}$ filtrate biomass) ${ }^{-1}$ ] where $x=$ product yield [g erythromycin (g producing biomass $)^{-1}$.

Antibiotic yield in the retentate $=\frac{C \cdot x}{C+D}$

[g erythromycin $(\mathrm{g} \text { retentate biomass })^{-1}$ ]

The units for the ratio of the specific production rates of the two fractions are:

$\frac{\text { g erythromycin (g filtrate biomass) })^{-1} h^{-1}}{\text { g erythromycin (g retentate biomass) }{ }^{-1} h^{-1}}$

This simplifies to:

g erythromycin ( $\mathrm{g}$ filtrate biomass) ${ }^{-1}$

$\overline{\mathrm{g} \text { erythromycin }(\mathrm{g} \text { retentate biomass) }}{ }^{-1}$

which can be obtained from the ratios of the expressions for productivity, above:

$$
=\frac{\left[\frac{A x}{A+B}\right]}{\left[\frac{C x}{C+D}\right]}
$$

This ratio is equal to $1 \cdot 29 / 1 \cdot 74=0.739$ (see specific production rates in preceding section).

At the minimum fragment diameter for production (assuming there is such a thing), $d_{\text {min }}$, the ratio of filtrate biomass greater than $d_{\min }$ to retentate biomass greater than $d_{\min }$ is equal to:

$$
\frac{A}{C}
$$

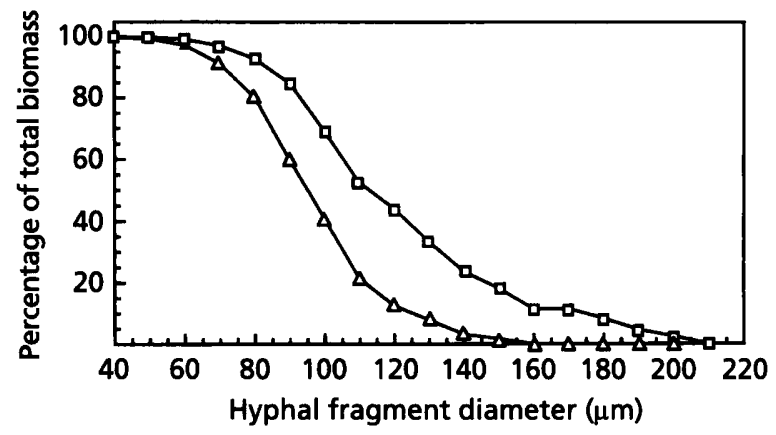

Fig. 5. The $y$-axis shows the proportion of hyphal fragments in the total population having a fragment size greater than that shown on the $x$ axis for filtrate $(\triangle)$ and retentate $(\square)$.

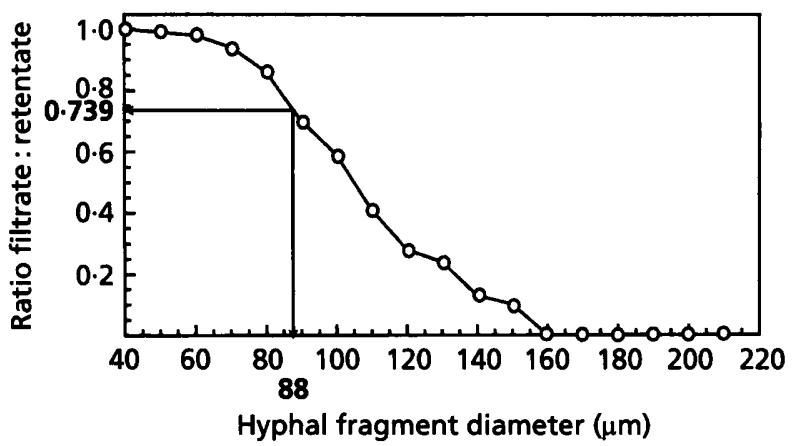

Fig. 6. Ratio of filtrate to retentate data shown in Fig. 5 as a function of fragment diameter.

Since $[A+B]=[C+D]=1 \mathrm{~g}$, expression (1) is equal to expression (2). Thus at the minimum diameter for production the ratio of filtrate: retentate biomass $=$ ratio of filtrate: retentate productivity $=0 \cdot 74$.

Checking this value against the corresponding hyphal fragment diameter (Figs 5 and 6) yields a diameter of $88 \mu \mathrm{m}$. Thus, if there is a minimum productive particle size, it is in the order of $88 \mu \mathrm{m}$, and below this size, productivity is insignificant.

\section{Variation in hyphal particle size during batch culture}

A bioreactor was sampled at intervals and filtration performed using the sintered glass filter. The proportion of retentate (larger particles, higher antibiotic production rate) to filtrate (smaller particles, lower antibiotic production rate) fraction varied with time (Fig. 7). The proportion of the less productive filtrate fraction ranged between $60 \%$ and $30 \%$, indicating that culture production potential is dependent on culture development. Dependence of branching rate on nutrient concentration (Bushell, 1988) may account for the variation in fragment size distribution with time if it is hypothesized that the hyphal branching rate influences susceptibility to breakage. The rise in proportion of the smaller particle size fraction between $60 \mathrm{~h}$ and $120 \mathrm{~h}$ may reflect the increased 


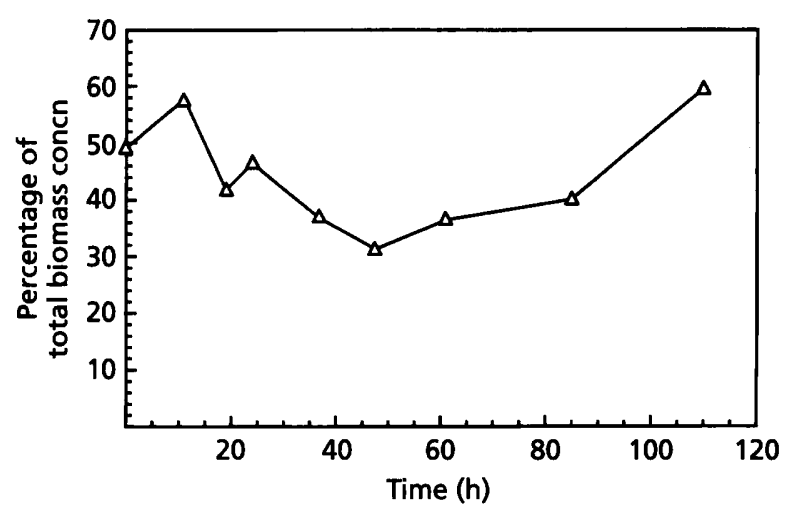

Fig. 7. Percentage of total biomass existing as filtrate (less productive fraction) as a function of time during nitrogenlimited batch culture.

appearance of unbranched hyphae (as a result of nutrient depletion) with greater susceptibility to breakage. Similarly, the overall decrease in proportion of the smaller particle size fraction during the early phase of the culture is consistent with a gradual increase in branching frequency (as a result of adaptation to relative nutrient abundance), leading to greater resistance to fragmentation. Alternatively, a mechanism such as that proposed by Paul et al. (1994), that mycelial breakage rate in fungi is dependent on the hyphal vacuolation rate induced by nutrient limitation, may also be operating in filamentous prokaryotes.

The observation (Fig. 7) that the proportion of bioreactor culture biomass capable of producing antibiotic varies significantly with time (assuming our hypothesis is correct) clearly has implications for commercial antibiotic production in terms of process economics. Our findings may also help to explain batch-to-batch variation in antibiotic production cultures, as the breakage susceptibility of inoculum cultures will vary according to nutrient availability and, probably, hyphal branching rate. These parameters are unlikely to be monitored at the time of inoculum transfer and our results indicate that they could influence antibiotic yield and productivity significantly.

\section{Influence of stirrer speed on particle size distribution and productivity}

In order to test the effect of stirrer speed on hyphal particle size distribution and productivity, chemostat cultures were operated at 1500 r.p.m. and 750 r.p.m. at a constant dilution rate $\left(0.08 \mathrm{~h}^{-1}\right)$. It has been reported that prolonged chemostat culture provides selective pressure for mutation to morphological variants in filamentous micro-organisms (Trinci, 1994). Experiments reported in the present paper were, therefore, replicated using fresh inocula to new cultures for each stirrer speed regime.

The particle size distribution was significantly affected by

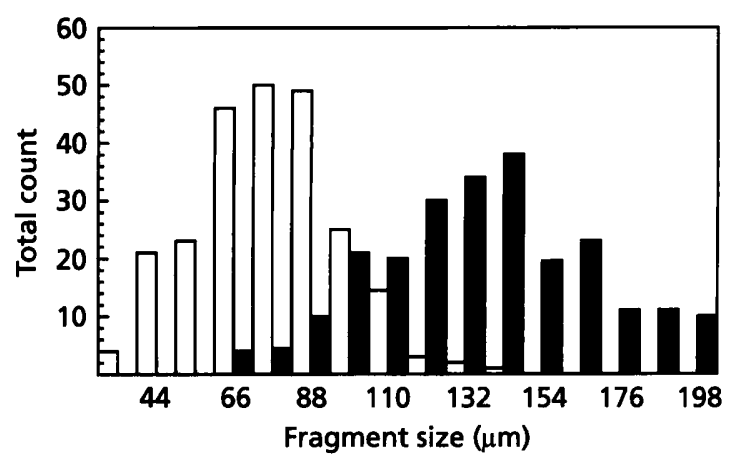

Fig. 8. Hyphal particle size distributions obtained during nitrogen-limited bioreactor culture at 1500 r.p.m. ( $\square$ ) and

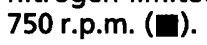

Table 2. Effect of stirrer speed on erythromycin productivity in a chemostat culture at $D=0.08 \mathrm{~h}^{-1}$ and statistical analysis of the hyphal particle size distribution data

$C=30 \cdot 315$, for the two sets of particle size data; therefore reject null hypothesis that there is no difference between particle size distribution at the two speeds $(P=0 \cdot 01)$.

\begin{tabular}{|c|c|c|}
\hline & \multicolumn{2}{|c|}{$\begin{array}{l}\text { Stirrer speed } \\
\text { (r.p.m.) }\end{array}$} \\
\hline & 750 & 1500 \\
\hline Biomass concn $\left(\mathrm{mg} \mathrm{ml}^{-1}\right)$ & $2 \cdot 04$ & $1 \cdot 92$ \\
\hline Erythromycin concn $\left(\mathrm{mg} \mathrm{l}^{-1}\right)$ & $23 \cdot 32$ & $20 \cdot 80$ \\
\hline $\begin{array}{l}\text { Specific antibiotic production rate } \\
\left(\mathrm{mg} \mathrm{g}^{-1} \mathrm{~h}^{-1}\right)\end{array}$ & 0.913 & 0.867 \\
\hline Sample size & 371 & 361 \\
\hline Skew $(\mu \mathrm{m})$ & 0.03 & $0 \cdot 20$ \\
\hline Sample mean $(\mu \mathrm{m})$ & $124 \cdot 01$ & $70 \cdot 13$ \\
\hline
\end{tabular}

stirrer speed (Fig. 8, Table 2), with a mean particle diameter of $70 \mu \mathrm{m}$ recorded at 1500 r.p.m. and $124 \mu \mathrm{m}$ recorded at 750 r.p.m. These observations are consistent with studies of growth and fragmentation in fungal bioreactor cultures which indicated that hyphal fragmentation rate is linearly related to the energy applied to the bioreactor, via the impeller (Shamlou et al., 1994; Nielsen \& Krabben, 1995). The specific antibiotic production rate responded accordingly (Table 2 ), with a higher erythromycin production rate $\left(0.913 \mathrm{mg} \mathrm{g}^{-1} \mathrm{~h}^{-1}\right)$ at 750 r.p.m. than at 1500 r.p.m. $\left(0 \cdot 867 \mathrm{mg} \mathrm{g}^{-1} \mathrm{~h}^{-1}\right)$. Since the experiment was carried out in chemostat culture, it was possible to eliminate most sources of difference between the two cultures other than particle size distribution. Thus, culture specific growth rate, dissolved oxygen and $\mathrm{pH}$ were identical in the two cultures and steady-state biomass concentration differed by less than $5 \%$.

These results are consistent with the hypothesis that there 


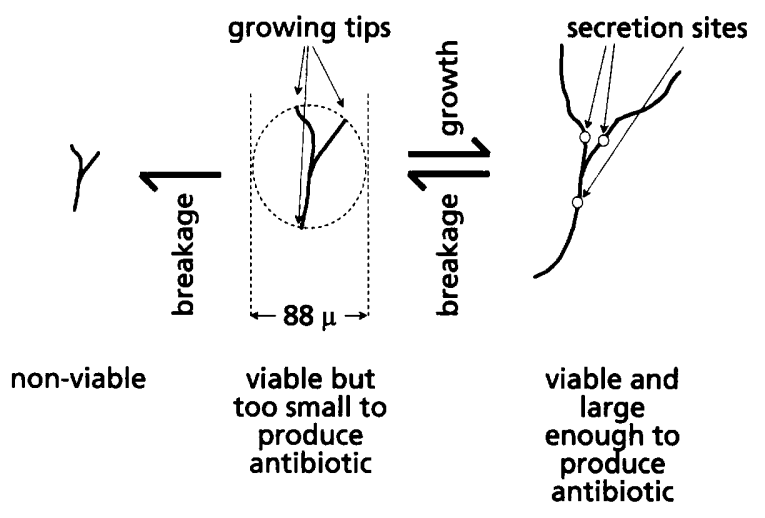

Fig. 9. Postulated relationship between hyphal elongation and growth in a stirred bioreactor culture, indicating the significance of the location of the antibiotic secretion sites.

is a critical hyphal fragment diameter $(88 \mu \mathrm{m})$, which must be exceeded if the fragment is to be able to produce antibiotic. This would be the case if the site of antibiotic secretion was located at a fixed distance behind the growing tip (Fig. 9). Particles below the critical size would grow but not produce antibiotic until they had increased in size sufficiently to include the hypothetical antibiotic secretion site. The concept of an antibiotic secretion site, at a fixed distance from the growing tip, has been hypothesized to explain the dynamics of antibiotic production in Penicillium chrysogenum cultures (Nestas \& Wang, 1983).

Hyphal breakage can also result in a decrease in viability (e.g. Jadubansa et al., 1994). Presumably, in a stirred bioreacter, the ability to produce antibiotic is influenced by a series of equilibria (Fig. 9). These will include the growth of hyphal particles from non-producing units (diameter below $88 \mu \mathrm{m}$ ) to larger antibiotic-producing particles, opposed by breakage into smaller viable, nonproducing units and, even smaller, non-viable units (Fig. $9)$.

\section{REFERENCES}

Bewick, M. W. \& Williams, S. T. (1977). Effects of addition of chloramphenicol on the growth and ultrastructure of Streptomyces venezuelae. Microbios 19, 27-35.

Bewick, M. W., Williams, S. T. \& Veltkamp, C. (1977). Growth and ultrastructure of Streptomyces venequalae during chloramphenicol production. Microbios 16, 191-199.

Bushell, M. E. (1988). Growth, metabolism and fermentation technology. In Biotechnology of the Actinomycetales, pp. 185-215. Edited by M. Goodfellow, S. T. Williams \& M. Mordarski. London: Academic Press.

Campbell, P. N. (1974). Statistics for Biologists. Cambridge: Cambridge University Press.

Huck, T. A., Porter, N. \& Bushell, M. E. (1991). Positive selection of antibiotic-producing soil isolates. J Gen Microbiol 137, 2321-2329.

Jadubansa, P., Lethbridge, G. L. \& Bushell, M. E. (1994). Physiology of production of viable biomass and spore inoculum for the biocontrol agent Idriella (Microdochium) bolleyi. Enzyme Microb Technol 16, 1-5.

Liu, Y., Yaun, C., Xu, M. \& Hu, J. (1984). Differentiation of Streptomyces kanamyceticus during industrial fermentation. Acta Microbiol Sin 24, 224-229.

Lynch, H. C. \& Bushell, M. E. (1995). The physiology of erythromycin biosynthesis in cyclic fed batch culture. Microbiology 141, 3105-3111.

McDermott, J. F., Lethbridge, G. \& Bushell, M. E. (1993). Estimation of the kinetic constants and elucidation of trends in growth and erythromycin production in batch and continuous cultures of Saccharopolyspora erytbraea using curve-fitting techniques. Enzyme Microb Technol 15, 657-663.

Nestas, E. \& Wang, D. I. C. (1983). Computer control of the penicillin fermentation using the filtration probe in conjunction with a structured process model. Biotechnol Bioeng 25, 781-796.

Nielsen, J. \& Krabben, P. (1995). Hyphal growth and fragmentation of Penicillium chrysogenum in submerged cultures. Biotechnol Bioeng 46, 588-598.

Packer, H. L. \& Thomas, C. R. (1993). Morphological measurements on filamentous microorganisms by fully automated image analysis. Biotechnol Bioeng 35, 870-871.

Paul, G. C., Kent, C. A. \& Thomas, C. R. (1994). Hyphal vacuolation and fragmentation in Penicillium chrysogenum. Biotechnol Bioeng 44, 655-660.

Pickup, K. M. \& Bushell, M. E. (1995). Non-fragmenting variants of Streptomyces hyphae have enhanced activity of an enzyme (phospho$\mathrm{N}$-acetylmuramyl pentapeptide translocase) in peptidoglycan biosynthesis. J. Ferment Bioeng 79, 247-251.

Pickup, K. M., Nolan, R. D. \& Bushell, M. E. (1993). A method for increasing the success rate of duplicating antibiotic activity in agar and liquid culture of Streptomyces isolates in new antibiotic screens. $J$ Ferment Bioeng 76, 89-93.

Roth, M., Noack, D. \& Reinhardt, G. (1982). Properties of nondifferentiating derivatives of Streptomyces bygroscopicus. J Gen Microbiol 128, 2687-2691.

Shamlou, P. A., Makagiansar, H. Y., Ison, A. P., Lilly, M. D. \& Thomas, C. R. (1994). Turbulent breakage of filamentous microorganisms in submerged culture in mechanically stirred bioreactors. Chem Eng Sci 49, 2621-2631.

Shomura. T., Yoshida, J., Amani, S., Kojima, M., Inouye, S. \& Niida, T. (1979). Studies on Actinomycetales producing antibiotics only on agar culture. 1. Screening, taxonomy and morphologyproductivity relationship of Streptomyces balstedii strain Sf-1993. J Antibiot 32, 427-435.

Trinci, A. P. J. (1994). Evolution of the Quorn mycoprotein fungus Fusarium graminearum A3/5. Microbiology 140, 2181-2188.

Tsuji, K. \& Goetz, J. F. (1978). High performance liquid chromatographic determination of erythromycin. J Chromatogr 147, 359-367.

Williams, S. T., Entwistle, S. \& Kurylowicz, W. (1974). The morphology of streptomycetes growing in media used for commercial production of antibiotics. Microbios 11a, 47-60.

Wilson, G. C. \& Bushell, M. E. (1995). The induction of antibiotic synthesis in Saccharopolyspora erythraea and Streptomyces bygroscopicus by growth rate down-regulation is accompanied by a downregulation in protein synthesis rate. FEMS Microbiol Lett 129, 89-96.

Received 11 October 1995; revised 22 January 1996; accepted 1 February 1996. 